\title{
The Advantages of the Signaling Strategy in a Dynamic Environment: Cognitive Modeling Using RoboCup
}

\author{
Sanjay Chandrasekharan ${ }^{1}$, Babak Esfandiari ${ }^{2}$, and Tarek Hassan ${ }^{2}$ \\ ${ }^{1}$ Institute of Cognitive Science \\ ${ }^{2}$ Department of Systems and Computer Engineering, \\ Carleton University, Ottawa, Canada, K1S 5B6 \\ \{schandra, babak, thassan\} @sce.carleton.ca
}

\begin{abstract}
We report a cognitive modeling experiment where the RoboCup simulation environment was used to study the advantages provided by signals. We used the passing problem in RoboCup as our test problem and soccerplayers' 'yells' of their 'passability' values as the task-specific signals. We found that yells improve pass completion - using yells to decide the best player (to pass the ball) led to a 8-17 percentage points increase in performance compared to a centralized calculation of best pass. However, the passability values themselves did not make a difference, indicating that the advantage of signals come from their different perspective in identifying a pass, the actual content of signals do not matter. We present some problems we faced in using Robocup as a modeling environment, and suggest features that would help promote the use of RoboCup in cognitive modeling.
\end{abstract}

\section{Introduction}

Many organisms generate stable structures in the world to reduce cognitive complexity, for themselves, for others, or both. Wood mice distribute small objects, such as leaves or twigs, as points of reference while foraging. They do this even under laboratory conditions, using plastic discs. Such "way-marking" diminish the likelihood of losing interesting locations during foraging [1]. Red foxes use urine to mark food caches they have emptied. This helps them avoid unnecessary search [2]. The male bower bird builds colorful bowers (nest-like structures), which females use to make mating decisions [3]. Such epistemic structures (ES), usually termed signals, are used widely, and form a very important aspect of animal life across biological niches. These structures allow organisms to hive off a part of their cognitive load to the world [5]. How much cognitive advantage do such structures provide in noisy, dynamic and adversarial environments? How robust is this advantage? What are its components? These are the problems addressed in this paper. We used RoboCup to study the cognitive advantages provided by the signaling strategy.

\section{Agent Design Taxonomy}

The section below develops a framework to understand how signaling (or the ES strategy, where the environment is changed in a way that it contributes task specific 
structures for decision-making), fits in with other agent-environment relationships. We categorize agent-world relations into four strategies, and use the design problem of providing disabled people access to buildings to illustrate these strategies.

Strategy 1: This involves building an all-powerful, James Bond-style vehicle that can function in all environments. It can run, jump, fly, climb spiral stairs, raise itself to high shelves, detect curbs etc. This design does not incorporate detailed environment structure into the vehicle, it is built to overcome the limitations of all environments.

Strategy 2: This involves studying the vehicle's environment carefully and using that information to build the vehicle. For instance, the vehicle will take into account the existence of short curbs, stairs being non-spiral and having rails, level of elevator buttons etc. So it will have the capacity to raise itself to short curbs, climb short flight of straight stairs by making use of the rails etc. Note that the environment is not changed here.

Strategy 3: This involves adding structure to the environment. For instance, building ramps and special doors so that a simple vehicle can have maximum access. This is the most elegant solution, and the most widely used one. Here structure is added to the environment, the world is "doped", so that it contributes to the agent's task.

Strategy 4: This strategy is similar to the first, but here the environment is allpowerful instead of the vehicle. The environment becomes "smart", and the building detects all physically handicapped people, and glides a ramp down to them, or lifts them up etc. This solution is an extreme case of strategy III, we will ignore it in the following analysis.

The first strategy is similar to the centralized AI one, which ignores the structure provided by specific environments. The environment is something to overcome, it is not considered a resource. This strategy tries to load every possible environment on to the agent, as centrally stored representations. The agent then tries to map the encountered world on to this internal template structure. The second strategy is similar to the situated AI model promoted by Rodney Brooks [6]. This strategy recognizes the role of the environment as a resource, and analyses and exploits the detailed structure that exists in the environment to help the agent. Notice the environment remains unchanged, it is considered a given. The third strategy is similar to one aspect of distributed cognition, where task-specific structures are generated in the environment, allowing the agent to hive off part of the computation to the world. Kirsh [7] terms this kind of "using the world to compute" active redesign. This principle is at work in the "intelligent use of space" where people organize objects around them in a way that helps them execute their functions [8].

\section{Using RoboCup to Study Epistemic Structure}

RoboCup provides an interesting dynamic and adversarial environment to study the efficiency of the ES strategy. However there is not much scope to add task-specific structure to the environment. The only structure that can be added is 'yells', or signals from teammates. We chose to use this structure, and studied the passing problem (i.e. how an agent in control of the ball can decide whom to pass the ball) to test the 
efficiency of this structure. We developed RoboCup teams that used three different approaches to passing. The teams were based on the UvA TriLearn 2002 code [9].

\subsection{Team 1: Centralized Passing}

This team (A1) uses approach 1 in our agent design taxonomy. A1 does not depend on task-specific information from other agents. In A1, when an agent has possession of the ball (i.e., the ball is within a kickable margin), it calculates the pass suitability (passability) for each teammate, and passes the ball to the teammate with the highest passability. If no teammate has passability above a fixed threshold value, the agent will dribble the ball toward the opponent goal. The goalie in this team is based on the original UvA algorithm, except for one modification: in a goal kick or free kick, the goalie will use A1 to calculate the best receiver for a pass and kick the ball to that teammate.

\subsection{Team 2: Passing with Yells}

This team (A2) is an implementation of the Active Design approach. Here every agent calculates its own passability. This calculation is done for every cycle a teammate has control of the ball. The fastest player in a set who can reach the ball is determined to have control of the ball. Once the passability value is calculated, each player uses the 'say' command to communicate this number to teammates. When updating the world model, every agent uses incoming aural messages from teammates to track the best passability at a given time. If a message arrives announcing a higher passability, then the sender of the message becomes the new best pass receiver. Every five cycles, the best passability is reset to the minimum threshold, to ensure that old information is not used to make the passing decision. As in centralized passing, the goalie uses A1 to calculate the pass receiver, but unlike its teammates, the goalie uses the centralized approach with no input from teammates. This ensures that the goalie always passes to someone.

\subsection{Team 3: Passing with Filtered Yells}

This team (A3) is also an implementation of Active Design, but it has some properties of the Brooksian approach, in the sense that it takes into consideration the limitation of the communication channel, which is a significant property of the environment. In A3, instead of agents saying their passability every cycle, agents listen to others' yells and compare their passability with the ones they hear. They announce their passability only if it is better. This lowers the load on the communication channel, by allowing only the best messages through. Once again, the goalie uses the centralized approach to passing.

\section{Experiments}

Each modified UvA team was pitted against the original UvA team to test the passing algorithms. Each team played 10 games. Logs of individual agents' decision-making were collected and analyzed to extract the successful and unsuccessful passes, and the 
passability values ${ }^{1}$. Note that even though A1 uses centralized decision-making to pass, the other agents in A1 calculate their own passabilities and store these values. In effect, all agents in all the three conditions calculate their passabilities when a teammate has the ball. In A2 and A3, this information was 'yelled', and the passing agent's decision to pass was based entirely on this information. In A1, there was no yelling by individual agents, they just stored their passability values, calculated in a centralized manner.

\section{Results}

\subsection{Pass Completion}

We analyzed the log files of games played by the three algorithms, and checked who next kicked or caught the ball after a player made a pass. If it was the intended recipient, the pass was completed, otherwise it failed. Table 1 shows the results of running the three modified teams against the original UvA team, and testing over ten games for each team.

Table 1. Number of passes completed

\begin{tabular}{llll}
\hline Team & Total Passes & Passes Completed & Percentage \\
\hline A1 & 2091 & 789 & $37.7 \%$ \\
A2 & 1534 & 401 & $28 \%$ \\
A3 & 3426 & 960 & $26.1 \%$
\end{tabular}

The number of passes are lower in the case of A2 than A1 because agents in A2 wait to hear yells, and if they don't hear a yell, they will dribble, instead of passing. The number of passes in A3 is higher in the case of A3 than A1 because A3 hears more yells. The above values show the performance of the three algorithms, given the narrow width of the communication channel (default, 1 message per cycle). Since the communication channel could not be broadened easily, we used two techniques to filter out the bottleneck effect of the communication channel, and capture the performance of the ES strategy better.

Technique 1: This involved potential receivers of the ball calculating their passabilities and logging them, even as the agent in control of the ball was calculating passabilities using the centralized algorithm. This means when an agent (say X) had control over the ball, all agents who could see $\mathrm{X}$ (say agents $\mathrm{C}, \mathrm{F}, \mathrm{G}, \mathrm{H}$ ) calculated their passabilities and stored their values. $X$ calculated its passability in a centralized fashion and logged that value. Our first filtering technique involved using these stored values to filter out only those passes where the agent in control of the ball (say X) decided to pass to the agent with the highest passability (say F) among the possible receivers, according to the estimates of the receivers. For example, let's say $\mathrm{X}$ had the ball and Agents $\mathrm{C}, \mathrm{F}, \mathrm{G}$ and $\mathrm{H}$ could see $\mathrm{X}$. In the first team (centralized algorithm, A1), $\mathrm{X}$ calculated the passabilities of agents C,F,G and $\mathrm{H}$ in a centralized manner. At

\footnotetext{
${ }^{1}$ We thank Neal Arthorne for implementing this algorithm and a log analysis tool.
} 
the same time, agents C,F,G and $\mathrm{H}$ calculated their own passability values with regard to $\mathrm{X}$. In A2 and A3 (the ES algorithms), $\mathrm{X}$ waited to hear the messages announcing passability from $\mathrm{C}, \mathrm{F}, \mathrm{G}$ and $\mathrm{H}$.

Consider the first case (algorithm A1). Let's say according to X's centralized calculation, $\mathrm{C}$ was the best pass. But according to the calculations of $\mathrm{C}, \mathrm{F}, \mathrm{G}$ and $\mathrm{H}$, agent $\mathrm{G}$ was the best pass. Here the passing agent and potential receivers disagree. But in some other instances, both the centralized calculation (passing agent) and the calculation by potential receivers agree (say they both calculate $\mathrm{C}$ as the best pass). Considering only passes of this latter kind is gives the same result as finding out situations where all the messages get through. The agreed situations pick out the instances where the passing agent decides to pass to the agent considered best by potential receivers. Note that this is true for all the three algorithms (A1, A2 and A3). This leads to a subset of the total passes being considered for analysis. These idealized passes (termed Agreed Passes) present the situation where the teammates' decision was communicated to the passing agent, and these passes incorporate their different perspective from the centralized agent. The following table presents the results from this analysis.

Table 2. Number of Completed Passes among Agreed Passes

\begin{tabular}{llll}
\hline Team & Total Passes & Passes Completed & Percentage \\
\hline A1 & 803 & 369 & $45.9 \%$ \\
A2 & 566 & 210 & $37.1 \%$ \\
A3 & 1536 & 518 & $33.7 \%$
\end{tabular}

These results show that the performance is significantly higher for agreed passes. This means receiving information from teammates (incorporating their perspective) leads to an increase in performance. There is an anomaly, however. The performance of A2 and A3 are still much below that of A1, with A2 barely matching A1's performance from the first analysis, and A3 performing 4 notches below that. Since agreement essentially takes away the limitations of communication, and considers only the scenarios where the signal is available to all the three algorithms, A2 and A3 should perform at least at the same level as A1, because they are all now using the same information (the potential receivers' assessment), and the same base level skills. Why is their performance lower?

One reason for the lower performance of A2 and A3 could be that the agents in control of the ball in A2 and A3 receive messages from potential receivers they can't see, like agents behind them, or at an angle to them. Passing to these invisible agents would be difficult, and the probability of such passes being completed is quite low. On the other hand, since A1 calculates only passabilities for agents it can see, agreed passes in A1 automatically leaves out agents it cannot see. This raises the power of its kicks and lowers randomness in the direction of the ball once it is kicked, raising the probability of completing the passes. This interaction between perspective and performance presents a trade-off in using the ES strategy in dynamic environments. On the one hand, the ES strategy can provide information from another perspective, and this is information an agent cannot get by using the centralized strategy. But on the other hand, given the physical limitations imposed by their perspectives, agents 
receiving this information may not be able to use it always. This means the ES strategy would be most effective in situations where the physical limitations of the agent are used to filter the information provided by the ES. To weed out the perspective-performance interaction, we analysed the data again from another angle.

Technique 2: This technique tries to filter out the perspective problem involved in a pass and focus entirely on the quality of information, i.e. the correlation between completion of passes and agreement. This is done by examining only the set of completed passes, and seeing how many of them were agreed passes. The chart below captures this.

Table 3. Number of agreed passes within completed passes

\begin{tabular}{llll}
\hline Team & Total Passes & Passes Completed & Percentage \\
\hline A1 & 789 & 369 & $46.7 \%$ \\
A2 & 401 & 210 & $52.3 \%$ \\
A3 & 960 & 518 & $53.9 \%$
\end{tabular}

The analysis shows that agreement predicts completion almost $48 \%$ of the time for A1, similar to the last analysis. This is expected, because A1 is not limited by perspective constraints, it calculates only those passes it can execute. On the other hand, the performance for A2 and A3 increases to around 52 and 54\%. If the passes can be executed well, the information provided by the ES strategy predicts completion better than the information provided by the centralized strategy (around 17 percentage points increase).

\subsection{Robustness Experiments}

To examine the robustness of the ES strategy, we manipulated two variables -- noise and time taken to calculate the pass. These variables approximated variations in the environment and the processing capabilities of organisms. The noise was varied by changing the player_rand parameter of the soccer server from 1 to 10 . The ES strategy outperformed the centralized strategy at all noise levels. The time taken to calculate the pass was varied by adding a sleep parameter to the passability function, and then varying the amount of sleep. The ES strategy once again performed better than the centralized decision-making strategy. These results show that the ES strategy is quite robust.

\subsection{Analysis of Passability Values}

From the previous analysis, we know that the passability calculation done by the potential receivers provides a better predictor of completion. But this analysis only compares the agent identified by the yelling agents and the passing agents (best passability), and not the passability values generated by the passing agent and the potential receivers. Is there a minimum value below which completion rates are low, and above which they are high? To understand this, we looked at the best passability values calculated by the yellers and the centralized agents, and then broke them down 
into 12 categories (10-20, 20-30 etc.), and looked at the total number of passes, completed passes and agreed\&completed passes. For a clearer picture on this, we looked at the total number of passes in each band of passability values (10-20, 20-30 etc.), and then looked at the average completion for each band. There was a slight increase in the completed passes as passability values increased, but the difference was not significant between the yells and centralized calculations. The similar completion rates (for yells and centralized) seem to indicate that the passability value does not differentiate between the two approaches (centralized, yells). One possible reason for this could be that the passability calculation is wrong, and does not make any difference at all. This can be ruled out, because agreement makes a significant difference in pass completion. A more plausible reason could be limitations imposed by the physical states of the agents (like stamina, view etc), which influences the strength of the kick, direction of the kick etc. The physical constraints set an upper limit to the completion of passes. The similar pattern of completion for different values, compared to the better completion rate seen in the earlier analysis for agreed passes, taken together indicate that having a higher passability value does not lead to better passes, but identifying the best agent makes a difference. That is, if both the yells and the centralized calculation identify agent $\mathrm{C}$ as the best pass, that improves the chance of the pass being completed. But yells or the centralized calculation deriving a 70-80 passability value for Agent $\mathrm{C}$ does not improve the chance of completion. This means the passability value is useful only for its relative perspective information, helping determine who is better among possible receivers. It is not a good indicator for pass completion. The ES strategy is better because of its perspective, and not because of its accuracy in calculating the passability value.

\section{Limitations and Future Work}

One of the major limitations of the study is the indirect way of assessing the effectiveness of the ES strategy. This is a direct result of the narrow communication channel. If the server parameters had allowed us to manipulate the hear_max value beyond 2 messages per cycle, we would've been able to judge the effectiveness of the strategy better. This would've also provided a way to better understand the relationship between channel-width and signal effectiveness in a dynamic environment. The freedom to change parameters, and a more user-friendly way of doing this, could lead to the RoboCup environment being used more widely by disciplines like cognitive science.

In this study, the opponent team was the same one for all the games. Even though this could be considered as providing a standardization for the results reported here, it is desirable to test a cognitive strategy in a variety of situations. Similarly, tests need to be done to determine the optimal number of waiting cycles used by a player in A2 and A3 before deciding on whom to pass. A further limitation is that the opponent team was not designed to intercept the passability messages, or to manipulate them. So the adversarial nature of the environment was limited to pass interception. In future work, we plan to use different teams against our teams. We also plan to 
investigate how unreliable messages affect the ES strategy. This is the equivalent of mimicry in biological systems.

\section{References}

1. Stopka, P. and Macdonald, D.W. Way-marking Behaviour: An Aid to Spatial Navigation in the Wood Mouse (Apodemus Sylvaticus). BMC Ecology,(2003)

2. Henry, J.D. The Use of Urine Marking in the Scavenging Behaviour of the Red Fox (Vulpes Vulpes). Behaviour, 62:82-105 (1977)

3. Zahavi, A. and Zahavi, A. The Handicap Principle: A Missing Piece of Darwin's puzzle. Oxford University Press, Oxford (1997)

4. Bradbury, J.W. \& Vehrencamp, S.L. Principles of Animal Communication. Sunderland, Mass: Sinauer Associates. (1998)

5. Chandrasekharan, S. \& Stewart, T.: Reactive agents learn to add epistemic structures to the world. In K. D. Forbus, D. Gentner \& T. Regier (Eds.), proceedings of the 26th Annual Meeting of the Cognitive Science Society, CogSci2004, Chicago. Hillsdale, NJ: Lawrence Erlbaum, (2004)

6. Brooks, R. A. Intelligence without Representation, Artificial Intelligence, 47:139-160. (1991)

7. Kirsh, D. Adapting the Environment Instead of Oneself. Adaptive Behavior, Vol 4, No. 3/4, 415-452. (1996)

8. Kirsh, D. The Intelligent Use of Space. Artificial Intelligence. 73: 31-68 (1995)

9. Kok, J.R. UvA TriLearn 2002 Source Code, University of Amsterdam (UvA), Faculty of Science Intelligent Autonomous Systems Group, (2002) 